ARTIGO ORIGINAL

\title{
Métodos da curva guia e equação das diferenças na classificação de sítio e sua relação na descrição da altura em Pinus taeda $L$.
}

\section{Guide curve and equation of differences methods in site classification and their relationship in the description of height in Pinus taeda L. stands}

\author{
Rodrigo Otávio Veiga de Miranda ${ }^{1}$ (1) , Afonso Figueiredo Filho ${ }^{2}$ (D), Emanuel Arnoni Costa ${ }^{1}$ (D), \\ Luan Demarco Fiorentin ${ }^{3}$ (D), Sintia Valerio Kohler ${ }^{4}$ (1), Ângelo Augusto Ebling ${ }^{4}$ (]) \\ ${ }^{1}$ Universidade Federal de Uberlândia - UFU, Monte Carmelo, MG, Brasil \\ ${ }^{2}$ Universidade Estadual do Centro Oeste - UNICENTRO, Irati, PR, Brasil \\ ${ }^{3}$ Universidade Federal do Paraná - UFPR, Curitiba, PR, Brasil \\ ${ }^{4}$ Universidade Federal Rural da Amazônia - UFRA, Parauapebas, PA, Brasil
}

Como citar: Miranda, R. O. V., Figueiredo Filho, A., Costa, E. A., Fiorentin, L. D., Kohler, S. V., \& Ebling, Â. A. (2021). Métodos da curva guia e equação das diferenças na classificação de sítio e sua relação na descrição da altura em Pinus taeda L. Scientia Forestalis, 49(131), e3565. https://doi.org/10.18671/scifor.v49n131.24

\begin{abstract}
Resumo
O objetivo deste trabalho foi gerar índices de sítio pelos métodos da curva guia e equação das diferenças e avaliar a influência nas estimativas de altura total para povoamentos de Pinus taeda L. Os dados foram provenientes de unidades amostrais permanentes de povoamentos não desbastados, localizados no município de Telêmaco Borba, estado do Paraná, com idade de 3,0 a 21,4 anos. Para a classificação da capacidade produtiva, o modelo de Chapman-Richards foi utilizado, considerando os métodos da curvaguia (CG) e equação das diferenças (ED). Os ajustes dos modelos foram avaliados pelo erro padrão da estimativa $\left(\mathrm{S}_{\mathrm{yx}} \%\right)$, correlação de Pearson $\left(r_{y \hat{y}}\right)$ e distribuição dos resíduos. Para verificar o padrão das curvas geradas, três testes para analisar o anamorfismo foram aplicados. Ainda, a estabilidade das unidades amostrais nas classes de índices de sítio foi verificada. Quatro modelos hipsométricos genéricos foram testados para estimar a altura total, no qual foi avaliada a influência da estimativa do índice de sítio na precisão desses modelos. Os modelos hipsométricos foram selecionados pelo coeficiente de determinação ajustado $\left(R^{2}{ }_{a j}\right)$, erro padrão da estimativa $\left(S_{y x} \%\right)$ e análise dos resíduos. Os dois métodos estimaram satisfatoriamente a altura dominante, contudo, as estimativas de índices de sítio diferiram estatisticamente. O método da ED promoveu maior estabilidade na classificação de sítio. Os testes para analisar o padrão das curvas indicaram que a relação altura dominante e idade podem ser representadas por curvas anamórficas. Os índices de sítio estimados por ambos os métodos de classificação de sítio, inseridos nos modelos hipsométricos, promoveram estimativas compatíveis estatisticamente e acuradas da altura total.
\end{abstract}

Palavras-chave: Índice de sítio; curvas anamórficas; relação altura-diâmetro.

\begin{abstract}
The objective of this work was to generate site indexes by different methods and to evaluate the influence on the estimates of total height in Pinus taeda $\mathrm{L}$ stands. The data came from permanent sampling units from non-thinned stands, located in the municipality of Telêmaco Borba, State of Paraná, aged 3.0 to 21.4 years old. For the classification of productive capacity, the Chapman-Richards model was used, considering the methods of the guide curve (CG) and equation of differences (ED). The fits of the models were evaluated by the standard error of the estimate $\left(\mathrm{S}_{\mathrm{yx}} \%\right)$, Pearson's correlation $\left(r_{y \hat{y}}\right)$ and distribution of residues. To verify the pattern of the generated site curves, three anamorphism tests were applied.
\end{abstract}

Fonte de financiamento: Nada a declarar.

Conflito de interesse: Nada a declarar.

Autor correspondente: rov_miranda@yahoo.com.br

Recebido: 26 junho 2020

Aceito: 6 abril 2021.

Editor: Paulo Henrique Müller Silva.

(c) (i) Este é um artigo publicado em acesso aberto (Open Access) sob a licença Creative Commons Attribution, que permite uso, distribuição e c) reprodução em qualquer meio, sem restrições desde que o trabalho original seja corretamente citado. 
Furthermore, the stability of the sampling units in the site index classes was verified. Four generic hypsometric models were tested to estimate the total height, in which the influence of the site index estimate on the precision of these models was evaluated. Hypsometric models were selected by the adjusted determination coefficient $\left(\mathrm{R}^{2}{ }_{\mathrm{aj}}\right)$, standard error of the estimate $\left(\mathrm{S}_{\mathrm{yx}} \%\right)$ and residual analysis. Both methods estimated the dominant height satisfactorily; however, the site index estimates differed statistically. The ED method promoted greater stability in the site classification. Tests to analyze the pattern of the curves indicated that the relationship between dominant height and age can be represented by anamorphic curves. The site indexes estimated by both methods of site classification, inserted in the hypsometric models, promoted statistically compatible and accurate estimates of the total height.

Keywords: Site index; anamorphic curves; height-diameter ratio.

\section{INTRODUÇÃO}

$\mathrm{O}$ ato de manejar um plantio florestal é sinônimo de gerenciá-lo, desde o planejamento até a comercialização de seus produtos. O manejador florestal faz uso de ferramentas específicas que geram informações essenciais à tomada de decisões, para caracterizar, quantificar e prognosticar o estoque de seus povoamentos com confiabilidade ao longo do tempo.

Para isso, algumas técnicas são frequentemente utilizadas, como o emprego de modelos estatísticos para estimar variáveis dendrométricas e a distinção de classes de produtividade ou sítio. O sítio é o espaço geográfico considerado homogêneo em relação às características físicas e biológicas (Skovsgaard \& Vanclay, 2008). Sua determinação é fundamental na definição das classes de produtividade e, consequentemente, para todo o sistema de prognose. O manejo de povoamentos florestais sem a distinção das classes de produtividade pode propiciar resultados indesejáveis (Téo et al., 2011).

O índice de sítio representa uma variável independente nos modelos de predição presente e futura do crescimento e da produção (Tonini et al., 2006) e importante fator para estratificação. Embora o sítio possa ser avaliado por métodos diretos e indiretos, a classificação direta é realizada em maior intensidade, especialmente a partir da relação da altura e idade de árvores indicadas como dominantes (Selle et al., 2008).

Dentre os métodos existentes para a construção de curvas de índice de sítio, o da curvaguia é o mais utilizado (Téo et al., 2011; Pego et al., 2015; Retslaff et al., 2015; Attis Beltrán et al., 2015), sendo considerado como padrão na comparação com outros métodos (Dias et al., 2005; Silva et al., 2016). Contudo, uma de suas limitações é a incapacidade de expressar tendências polimórficas.

No entanto, outros métodos podem ser utilizados na construção de curvas de índices de sítio. Dentre estes, tem-se o de Hammer (1981), predição dos parâmetros (Dias et al., 2005; Campos \& Leite, 2017), equação das diferenças ou diferença algébrica (Dias et al., 2005; Castro et al., 2015; Silva et al., 2016; Santos et al., 2018) e método da diferença algébrica generalizada (GADA) (Nunes et al., 2011; Akbas \& Senyurt, 2018).

Devido à existência de diferentes métodos, alguns estudos foram conduzidos para avaliar a compatibilidade entre as estimativas de índices de sítio. Contudo, tão importante como avaliar essa similaridade, é verificar o efeito da estimativa do sítio como variável independente na modelagem de árvores ou para o povoamento. Neste sentido, duas hipóteses foram avaliadas neste trabalho, sendo $h_{0(1)}$ : os métodos fornecem estimativas de índice de sítio estatisticamente compatíveis; $h_{0(2)}$ : a precisão de estimativas de altura total com modelos hipsométricos não é afetada pelo método utilizado para gerar estimativas de índice de sítio. Diante do exposto, o objetivo deste trabalho foi gerar índices de sítio pelo método da curva guia e da equação das diferenças e analisar a influência nas estimativas de altura total para povoamentos não desbastados de Pinus taeda L. 


\section{MATERIAL E MÉTODOS}

\section{Área de estudo}

A área em que foi realizado este trabalho está localizada na região dos Campos Gerais, município de Telêmaco Borba, Paraná, com relevo suavemente ondulado e altitude média de $850 \mathrm{~m}$ acima do nível do mar. De acordo com a classificação de Köppen-Geiger, o clima do local é do tipo $\mathrm{Cfa}$, com temperatura média anual de $18,4{ }^{\circ} \mathrm{C}$ e pluviosidade média anual acumulada de $1.378 \mathrm{~mm}$ (Climate-Data.Org, 2021).

Os dados foram provenientes de unidades amostrais permanentes distribuídas aleatoriamente em povoamentos de Pinus taeda L. não desbastados, plantados no arranjo de 2,5 m x 2,5 m. A intensidade amostral foi de, aproximadamente, uma unidade amostral por hectare. Cada unidade amostral foi delimitada por 10 linhas de plantio e 10 plantas em cada linha.

Em cada unidade amostral, os diâmetros à altura do peito de todas as árvores, altura total (h) das 10 primeiras e das árvores dominantes conforme metodologia proposta por Assmann (1970) foram medidos. No total, 2.396 unidades amostrais foram utilizadas, com intervalo de remedição de 1, 2, 3 ou 4 anos, com idade variando de 3,0 a 21,4 anos.

\section{Determinação das classes de índices de sítio}

A determinação das classes de capacidade produtiva foi realizada empregando o método direto baseado na relação da altura e idade de árvores dominantes, por meio da definição de índices de sítio. O modelo de Chapman-Richards foi utilizado, sendo amplamente indicado no meio florestal e capaz de gerar estimativas acuradas (Retslaff et al., 2015; Santos et al., 2018; Schuchovski et al., 2019).

Além disso, esse modelo possui flexibilidade para demonstrar vários tipos de tendências e propriedades que garantem representar satisfatoriamente o crescimento típico biológico (Zeide, 1993), sendo expresso como: $h_{\text {dom }}=\beta_{0}\left(1-e^{-\beta_{I} I}\right)^{\beta_{2}}+\varepsilon_{i}$, em que $h_{\text {dom }}$ é a altura dominante da unidade amostral (m), I representa a idade (anos), $\beta_{i}$ são os parâmetros a serem estimados por regressão não linear, "e" é o exponencial natural $(2,718282)$ e $\varepsilon_{i}$ é o erro aleatório do modelo para a estimativa $i$, em que $i=1,2, \ldots, n$, sendo $n$ é o número de observações.

Para gerar as curvas de índices de sítio, os métodos da curva-guia (CG) e equação das diferenças (ED) foram utilizados. No método da CG, a equação ajustada representa a tendência média dos valores de altura dominante $\left(h_{\text {dom }}\right)$ observados ao longo do tempo. A partir da definição da idade de referência $\left(I_{\text {ref }}\right)$, os valores de $h_{\text {dom }}$ nessa idade (I) corresponde ao índice de sítio (S), ou seja, $h_{\text {dom }}=\mathrm{S} \leftrightarrow \mathrm{I}=\mathrm{I}_{\text {ref. }}$ Considerando-se o modelo de ChapmanRichards, a relação funcional $h_{\text {dom }}=S\left[\left(1-e^{-\hat{\beta}_{I} I}\right) /\left(1-e^{-\hat{\beta}_{I} I_{r e f}}\right)\right]^{\hat{\beta}_{2}}$ foi utilizada para gerar as curvas limites de índice de sítio (Retslaff et al., 2015), em que $\hat{\beta}_{1}$ e $\hat{\beta}_{2}$ são os coeficientes do modelo, $\mathrm{I}_{\text {ref }}$ representa a idade de referência (18 anos), "S", "h $\mathrm{h}_{\mathrm{dom}}$ ", "I" e "e" como mencionados anteriormente.

No método da equação das diferenças, o modelo é ajustado considerando o pareamento dos valores de $h_{\text {dom }}$ e I. Uma das vantagens deste método é a possibilidade de construção de curvas anamórficas e polimórficas, o que dependerá do conceito utilizado na obtenção da expressão para gerar as curvas de sítio. Devido a quase totalidade das unidades amostrais possuírem apenas duas medições, a expressão a ser submetida ao ajuste foi a utilizada para a construção de curvas anamórficas (Santos et al., 2018), dada por $h_{\text {dom }_{2}}=h_{\text {dom }_{l}}\left[\left(1-e^{-\beta_{I} I_{2}}\right) /\left(1-e^{-\beta_{I} I_{I}}\right)\right]^{\beta_{2}}+\varepsilon_{i}$, em que $h_{\text {dom1 }}$ e $h_{\text {dom2 }}$ representam a altura dominante (m) na idade anterior $\left(\mathrm{l}_{1}\right)$ e posterior $\left(\mathrm{I}_{2}\right)$ (anos), respectivamente, $\beta_{i}$, "e" e $\varepsilon_{i}$ como mencionados anteriormente. Após o ajuste, a relação funcional para gerar as curvas de sítio foi obtida considerando a $I_{1}$ como a idade de referência e, consequentemente, a $h_{d o m_{l}}$ como o $\mathrm{S}$. 
Conforme a variação da altura dominante nas várias idades, três classes de sítio foram definidas para ambos os métodos, sendo as classes I, II e III, representando os sítios de alta, média e baixa produtividade. A amplitude entre as curvas foi de $5 \mathrm{~m}$.

Os ajustes dos modelos foram realizados por regressão não linear e avaliados pelo erro padrão da estimativa $\left(S_{y x} \%\right)$, correlação de Pearson $\left(r_{y \hat{y}}\right)$ entre os valores observados e estimados e distribuição dos resíduos. A significância estatística utilizada nestas análises foi de $5 \%(a \leq 0,05)$.

Curvas de índices de sítio foram construídas para cada método avaliado, sendo verificado o comportamento das curvas perante os valores observados. O teste "t" pareado $(\alpha \leq 0,05)$ foi aplicado para avaliar a hipótese de igualdade dos valores estimados de índice de sítio pelos métodos sob avaliação. Ainda, o percentual de alternância entre classes de sítio foi analisado.

Para verificar o anamorfismo ou polimorfismo das curvas de índices de sítio geradas pelos métodos em estudo, três testes de anamorfismo foram aplicados. O primeiro teste se baseou no cálculo do coeficiente de variação em altura dominante média por idade e classe de sítio. Uma das pressuposições básicas do anamorfismo é que esses valores sejam semelhantes em diferentes idades para uma mesma classe de sítio (Scolforo, 2006).

O segundo teste foi baseado na relação linear entre o índice de sítio e a altura dominante média em diferentes idades, $S=\beta_{0}+\beta_{1} h_{d o m}+\varepsilon_{i}$, demonstrando que o índice de sítio não depende da idade, mas do potencial produtivo do local (Kirby, 1975). O esperado é que sejam obtidos valores de intercepto $\left(\hat{\beta}_{0}\right)$ e de coeficiente angular $\left(\hat{\beta}_{1}\right)$ estatisticamente iguais e diferentes de zero, respectivamente (Scolforo, 2006). Além disso, que o coeficiente angular reduza com o avanço da idade, e admita valores maiores que um, igual a um e menor que um, nas idades menores, igual e maiores à idade de referência, respectivamente.

O terceiro teste é abordagem gráfica proposta por DeMars (1969). Primeiramente, é necessário obter a razão entre as alturas dominantes médias na idade de referência para cada classe de sítio, em relação à altura dominante média do limite inferior da classe de menor produtividade. Após, deve-se multiplicar cada altura dominante ao longo do tempo pela respectiva razão encontrada para a classe de sítio, e apresentadas graficamente. Conforme o autor, a sobreposição das curvas ou pequenos desvios entre elas confirma o anamorfismo.

\section{Modelagem hipsométrica}

As estimativas de índice de sítio para os métodos sob análise foram utilizadas como variável independente em modelos hipsométricos. Para os ajustes desses modelos, dados de 1.356 unidades amostrais foram utilizados, com idades de 3,2 a 21,0 anos. No total, 33.877 alturas foram usadas, em que $80 \%$ foram empregadas no ajuste e $20 \%$ na validação das equações, selecionado proporcionalmente em relação às classes de sítio.

Objetivando obter uma equação genérica, modelos hipsométricos em função de variáveis que expressam o tamanho, idade e qualidade produtiva foram testados (Tabela 1). Para o ajuste dos modelos lineares, o método dos mínimos quadrados ordinários foi empregado. Para o modelo (4), único não linear, o algoritmo de Levenberg-Marquardt foi utilizado.

Tabela 1. Modelos genéricos para estimar altura total de um povoamento de Pinus taeda $\mathrm{L}$.

\begin{tabular}{ccc}
\hline $\mathbf{N}^{\circ}$. Modelo & Autor/Fonte & Modelo \\
\hline 1 & Campos \& Leite (2017) & $h=\beta_{0}+\beta_{1} d^{-1}+\beta_{2} S+\beta_{3} \ln (I)+\varepsilon_{i}$ \\
2 & Campos \& Leite (2017) & $h=\beta_{0}+\beta_{1} S+\beta_{2} \ln (I d)+\beta_{3} I+\varepsilon_{i}$ \\
3 & Nogueira (2003) & $h=\beta_{0}+\beta_{I} I+\beta_{2} d+\beta_{3} S+\varepsilon_{i}$ \\
4 & Nogueira (2003) & $h=e^{\beta_{0}+\beta_{l} S+\beta_{2} d^{-1}+\beta_{3} \ln (I)}+\varepsilon_{i}$
\end{tabular}

Em que: $\mathrm{h}$ = altura total $(\mathrm{m}) ; \mathrm{d}=$ diâmetro à altura do peito $(\mathrm{cm}) ; \mathrm{S}$ = índice de sítio $(\mathrm{m}) ; \mathrm{I}=$ idade (anos); e = exponencial natural (2,718282); $\widehat{\beta}_{\mathrm{i}}=$ coeficientes a serem estimados; $\varepsilon_{\mathrm{i}}=$ erro aleatório do modelo para a estimativa $\mathrm{i}$, em que $\mathrm{i}=1$, $2, \ldots, \mathrm{n}$, sendo $\mathrm{n}$ é o número de observações. 
As estatísticas utilizadas para avaliar os modelos foram o coeficiente de determinação ajustado $\left(R^{2}{ }_{a j}\right)$, erro padrão da estimativa percentual $\left(S_{y \times} \%\right)$ e análise gráfica dos resíduos (Draper \& Smith, 1998). A compatibilidade das estimativas de altura total dos melhores modelos para os métodos da CG e ED foi averiguada pela análise gráfica associada à correlação de Pearson $\left(r_{y \hat{y}}\right)$. A avaliação da precisão dos modelos aplicados à base de dados selecionada para validação foi dada pela análise da raiz do quadrado médio do resíduo

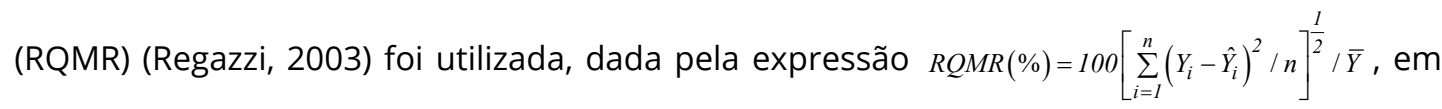
que RQMR foi calculada em percentagem, sendo " $\mathrm{n}$ " o número de pares de dados, $Y_{i}$ e $\hat{Y}_{i}$ os valores observados e estimados, respectivamente. O nível de significância adotado na regressão e para a significância dos coeficientes foi de $5 \%$.

Para o melhor modelo, curvas de crescimento em altura em diferentes idades foram elaboradas. Esta análise foi realizada para cada classe de índice de sítio.

\section{RESULTADOS E DISCUSSÃO}

\section{Determinação e avaliação das classes de índices de sitio}

O modelo de Chapman-Richards foi ajustado, gerando coeficientes significativos para os dois métodos em estudo. Para o método da curva-guia, a equação $h_{\text {dom }}=41,224399\left(1-e^{-0,061832 I}\right)^{1,156161}$ resultou nos valores de $\mathrm{S}_{\mathrm{yx}}$ de $9,56 \%$ e $r_{y \hat{y}}$ de 0,97 . Para o método da equação das diferenças, a equação resultante foi $h_{\text {dom }}=h_{\text {dom }}\left[\left(1-e^{-0,069320 I_{2}}\right) /\left(1-e^{-0.069320 I_{I}}\right)\right]^{1,093267}$, proporcionando estatísticas comparativamente superiores, com valores de $\mathrm{S}_{\mathrm{yx}}$ e $r_{\hat{y \hat{y}}}$ de 6,38\% e 0,98, respectivamente.

A distribuição residual evidencia a superioridade estatística do método da ED (Figura 1). Para esse método, a distribuição dos resíduos apresentou-se com maior simetria e menor amplitude de variação, concentrados em $\pm 21 \%$. Para o método da $C G$, a distribuição dos resíduos indica menor precisão das estimativas de $h_{\text {dom }}$ em relação ao método da $E D$, com sutil tendência de subestimar a $h_{\text {dom, }}$ além de contemplar maior amplitude, cerca de $\pm 33 \%$.
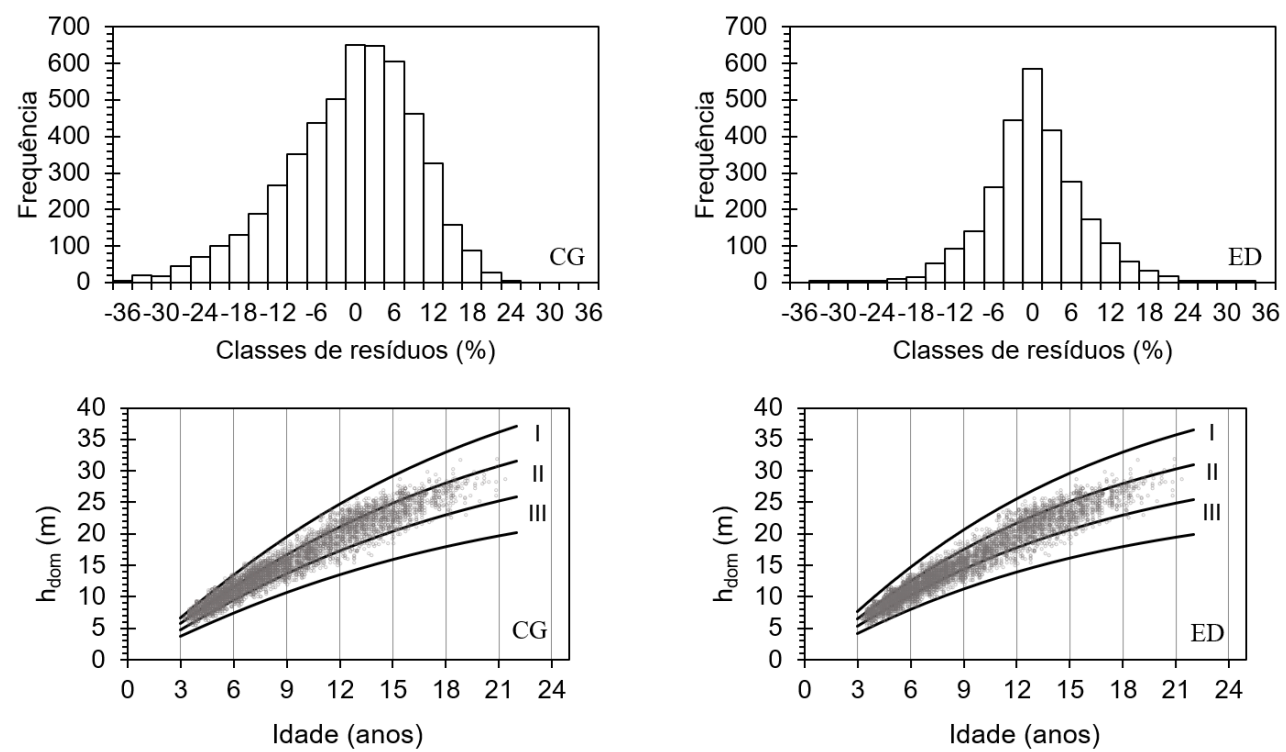

Figura 1. Distribuição residual para a relação da altura dominante e idade, para o modelo de ChapmanRichards e curvas de índice de sítio para o método da curva-guia (CG) e equação das diferenças (ED), para um povoamento de Pinus taeda L. 
Esse melhor resultado do método da ED pode ser atribuído à menor variabilidade da variável dependente do modelo ajustado. A necessidade de pareamento das informações para o ajuste da expressão deste método promoveu redução do coeficiente de variação da $\mathrm{h}_{\mathrm{dom}}$, de $37 \%$ para $32 \%$, o que refletiu nas estatísticas obtidas.

Ao analisar as curvas de índice de sítio geradas pelos métodos avaliados (Figura 1), ambos descreveram satisfatoriamente a tendência observada de altura dominante em função da idade, produzindo curvas bem semelhantes. Os valores de índice de sítio gerados pelo método da ED foram ligeiramente maiores e menores nas idades inferiores a superiores à idade de referência, respectivamente. Essas diferenças aumentaram à medida que se aumentou o índice de sítio. Embora essas diferenças sejam visualmente de baixa magnitude, o teste " $\mathrm{t}$ " pareado indicou a existência de diferença estatística entre os limites das classes de índices de sítio gerados pelos métodos em análise, com p-valores inferiores a 0,01.

Três classes de produtividade foram consideradas, sendo a classe I compreendendo alturas dominantes na idade de referência de 28,1 a 33,0 m; a classe II englobando alturas dominantes entre 23,1 a 28,0 m; e a classe III envolvendo alturas dominantes de 18,0 a 23,0 $\mathrm{m}$, representando a classe de produtividade alta, média e baixa, respectivamente.

Por meio da relação funcional para gerar as curas de sítio específica para cada método, o S para cada unidade amostral foi obtido e, conforme esse valor, as unidades amostrais foram alocadas às respectivas classes. Analisando a alternância das unidades amostrais nas diferentes classes de sítio (Figura 2), o método da ED apresentou maior estabilidade, no qual $91 \%$ das unidades amostrais permaneceram na mesma classe em diferentes idades, e somente $6 \%$ e $2 \%$ oscilaram entre as classes de sítio II e III e I e II, respectivamente. No método da CG, ocorreu oscilação maior, com destaque à alternância entre as classes de sítio I e II (17\%). Para este método, o percentual sem alternância foi de $72 \%$.

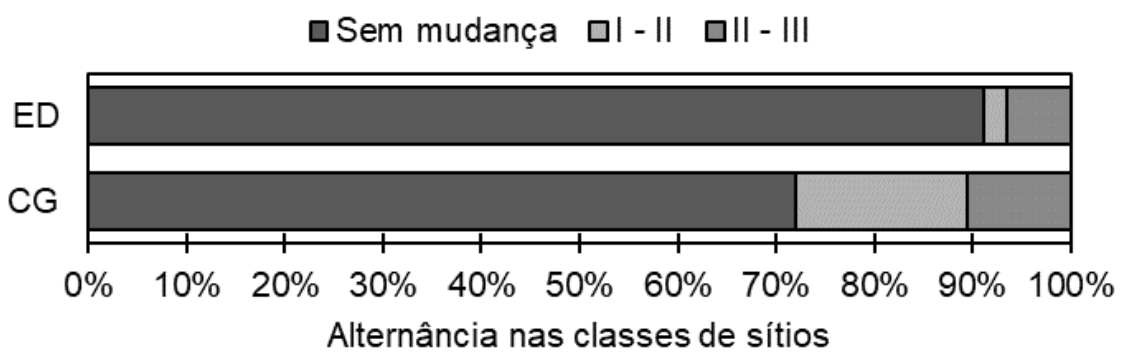

Figura 2. Estabilidade das unidades amostrais nas classes de índices de sítios geradas pelos métodos da curva-guia (CG) e equação das diferenças (ED), para um povoamento de Pinus taeda $\mathrm{L}$.

A instabilidade de unidades amostrais nas classes de índices de sítio se concentrou em grande parte nas idades iniciais. Aproximadamente, $70 \%$ dos problemas de instabilidade foram observados em idades inferiores aos oito anos. Essa instabilidade inicial é provavelmente ocasionada pela adaptação da planta ao local de crescimento nas idades mais jovens (Tonini et al., 2006). Portanto, a utilização destas curvas em povoamentos jovens pode promover classificação inadequada das classes de produtividade (Selle et al., 1994; Machado et al., 1997).

Unidades amostrais que se encontram próximas aos limites da classe de sítio podem flutuar de um sítio para outro em função de alguma mudança climática ou outro fator do meio que influencie seu desenvolvimento (Silva et al., 2013). Schuchovski et al. (2019) também observaram alternância entre classes de sítio para algumas unidades amostrais, em classificação realizada para povoamentos de Pinus taeda L. localizados nos estados do Paraná, Santa Catarina e São Paulo, com idades de 3,7 a 33,2 anos.

Ao construir curvas de índice de sítio por meio do método da CG para plantios de Tectona grandis L. f. nos municípios de Rio Branco e Acrelândia, estado do Acre, Figueiredo (2005) observou estabilidade de $56,7 \%$ das unidades amostrais nas classes de sítio. Tonini et al. 
(2006) também detectaram oscilações de unidades amostrais entre classes de índice de sítio, em classificação pelo método da CG para plantios clonais de Eucalyptus saligna Smith para a Depressão Central e Serra do Sudeste, Estado do Rio Grande do Sul.

É importante destacar que esses resultados podem ser influenciados pela natureza dos dados deste estudo. Como mencionado, grande parte das unidades amostrais, cerca de $70 \%$, possuem apenas uma medição e/ou uma remedição, o que impede o acompanhamento do crescimento da $\mathrm{h}_{\text {dom }}$ ao longo do desenvolvimento do povoamento. O percentual restante, cerca de $30 \%$, possui duas remedições.

$\mathrm{Na}$ análise do anamorfismo e/ou polimorfismo das curvas de índices de sítios, os coeficientes de variação foram baixos em todas as situações (Tabela 2). Esses valores indicam o anamorfismo das curvas de índice de sítio construídas, dada à semelhança nas diversas idades para uma mesma classe de sítio, corroborado com resultados de Scolforo (1992) e Téo et al. (2011). De maneira geral, a variação foi menor para o método da equação das diferenças.

Tabela 2. Coeficiente de variação da altura dominante por idade e classe de índice de sítio geradas pelos métodos da curva-guia (CG) e equação das diferenças (ED), para um povoamento de Pinus taeda L.

\begin{tabular}{|c|c|c|c|c|c|c|c|c|c|c|c|c|c|c|c|c|c|c|c|c|}
\hline \multirow{2}{*}{ Método } & \multirow{2}{*}{$S$} & \multicolumn{19}{|c|}{ Idade (anos) } \\
\hline & & 3 & 4 & 5 & 6 & 7 & 8 & 9 & 10 & 11 & 12 & 13 & 14 & 15 & 16 & 17 & 18 & 19 & 20 & 21 \\
\hline \multirow{3}{*}{ CG } & 1 & 3,5 & 4,2 & 3,9 & 4,2 & 3,8 & 4,0 & 3,5 & 2,9 & 5,7 & 3,2 & 2,8 & 1,8 & 2,2 & 2,1 & 2,6 & 3,0 & - & - & - \\
\hline & II & 2,5 & 5,5 & 5,4 & 6,0 & 5,2 & 5,3 & 4,8 & 5,2 & 4,7 & 5,1 & 5,2 & 5,8 & 4,6 & 5,7 & 3,0 & 5,7 & 4,6 & 5,4 & 5,6 \\
\hline & III & - & 5,5 & 4,8 & 4,4 & 4,4 & 3,2 & 4,3 & 4,3 & 2,9 & 5,7 & 3,6 & 3,1 & 4,1 & 2,0 & 1,5 & 1,3 & - & - & - \\
\hline \multirow{3}{*}{ ED } & 1 & - & 1,9 & 1,5 & 2,5 & 1,9 & 1,3 & 1,5 & 2,3 & 3,8 & 2,6 & 2,2 & 1,4 & 1,3 & 0,8 & 2,6 & 3,0 & - & - & - \\
\hline & II & 4,1 & 6,7 & 5,9 & 5,1 & 5,2 & 5,2 & 4,5 & 5,3 & 4,9 & 5,9 & 5,4 & 5,7 & 4,8 & 5,6 & 0,8 & 5,7 & 5,9 & 6,2 & - \\
\hline & III & - & 7,5 & 6,5 & 5,8 & 6,1 & 4,1 & 5,5 & 5,7 & 5,7 & 5,7 & 4,1 & 4,4 & 4,1 & 1,4 & 1,0 & 0,1 & - & - & - \\
\hline
\end{tabular}

Em que: $\mathrm{S}$ = índice de sítio $(\mathrm{m}) ; \mathrm{CG}=$ método da curva-guia; $\mathrm{ED}=$ método da equação das diferenças.

O anamorfismo e/ou polimorfismo das curvas de índices de sítio foi verificado pelo ajuste do modelo linear para os métodos da CG e ED. Na Figura 3a está apresentado apenas o resultado deste teste para o método da CG, em função da grande semelhança com aqueles para o método da ED. Para o coeficiente $b_{0}$ (intercepto), os $p$-valores foram todos acima do nível de significância selecionado, com valores iguais ou próximos a zero, indicando a não rejeição da hipótese de nulidade da regressão linear $\left(\beta_{0}=0\right)$.
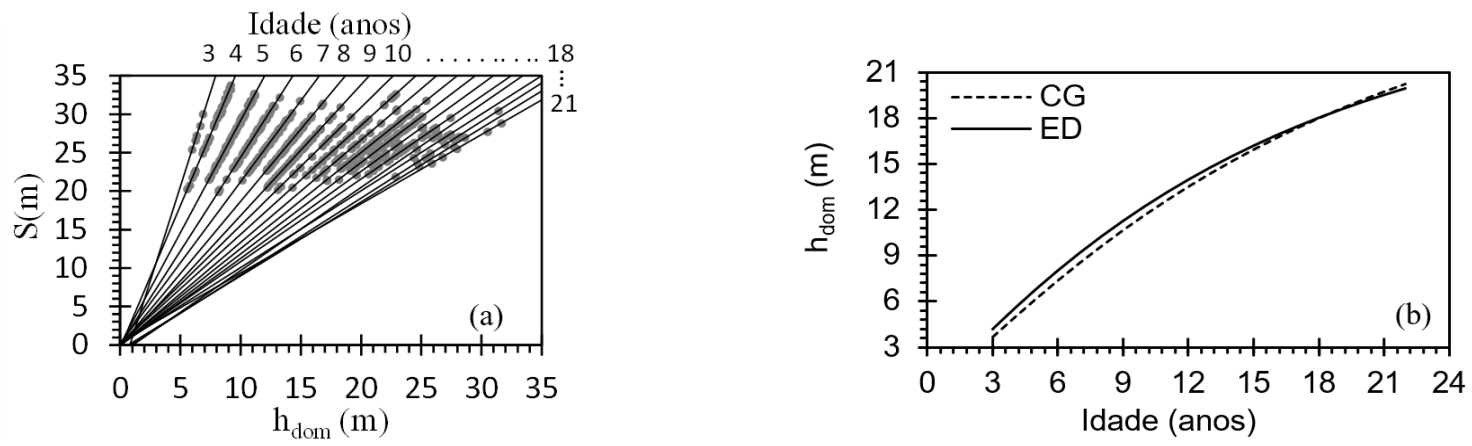

Figura 3. Correlação entre índice de sítio obtido pelo método da curva-guia e altura dominante em diferentes idades (a) e curvas de índice de sítio após aplicação do teste de anamorfismo de DeMars para os métodos da curva-guia (CG) e equação das diferenças (ED), para um povoamento de Pinus taeda L. 
Tabela 3. Coeficientes e estatísticas de avaliação dos modelos testados para estimar altura total em um povoamento de Pinus taeda $\mathrm{L}$.

\begin{tabular}{|c|c|c|c|c|c|c|c|c|}
\hline \multirow{2}{*}{ Método } & \multirow{2}{*}{ Modelo } & \multirow{2}{*}{$\widehat{\boldsymbol{\beta}}_{0}$} & \multirow{2}{*}{$\widehat{\boldsymbol{\beta}}_{1}$} & \multirow{2}{*}{$\widehat{\boldsymbol{\beta}}_{2}$} & \multirow{2}{*}{$\widehat{\boldsymbol{\beta}}_{3}$} & \multicolumn{2}{|c|}{ Ajuste } & \multirow{2}{*}{$\begin{array}{l}\text { Validação } \\
\text { RQMR (\%) }\end{array}$} \\
\hline & & & & & & $\mathbf{R}^{2}{ }_{\text {aj }}$ & $S_{y x} \%$ & \\
\hline \multirow{4}{*}{ CG } & 1 & $-10,78807$ & $-64,12279$ & 0,25196 & 10,60151 & 0,91 & 11,38 & 11,68 \\
\hline & 2 & $-19,33847$ & 0,18481 & 4,66582 & 0,59133 & 0,93 & 9,87 & 9,92 \\
\hline & 3 & $-3,78425$ & 1,02774 & 0,27619 & 0,12453 & 0,93 & 9,85 & 9,70 \\
\hline & 4 & 1,21133 & 0,01048 & $-6,34359$ & 0,69040 & 0,94 & 9,40 & 9,16 \\
\hline \multirow{4}{*}{ ED } & 1 & $-9,83849$ & $-64,14465$ & 0,27197 & 10,10742 & 0,91 & 11,34 & 11,63 \\
\hline & 2 & $-18,92164$ & 0,20058 & 4,62128 & 0,55976 & 0,93 & 9,88 & 9,91 \\
\hline & 3 & $-3,71111$ & 1,00152 & 0,27559 & 0,14018 & 0,93 & 9,82 & 9,65 \\
\hline & 4 & 1,25243 & 0,01123 & $-6,34401$ & 0,66995 & 0,94 & 9,39 & 9,13 \\
\hline
\end{tabular}

Em que: $\mathrm{R}^{2}{ }_{\mathrm{aj}}=$ coeficiente de determinação ajustado; $\mathrm{Syx}_{\mathrm{y}} \%$ = erro padrão da estimativa percentual; $\mathrm{RQMR}(\%)=$ raiz do quadrado médio do resíduo percentual; $\hat{\beta}_{\mathrm{i}}=$ coeficientes estimados.

Para o coeficiente $\beta_{1}$ (coeficiente angular), os p-valores confirmaram a rejeição da hipótese de nulidade para todos os casos. $O$ coeficiente $b_{1}$ admitiu valores maiores, igual $e$ menor que um, nas idades menores, igual e maiores à idade de referência, respectivamente. Em todos os casos, o $\mathrm{R}^{2}$ produziu valores iguais ou muito próximos a um.

A tendência de redução dos valores dos coeficientes angulares era esperada. Nas classes iniciais de idade, a amplitude entre as curvas de sítio é menor, de modo que pequenas alterações na altura dominante podem representar migrações entre as classes de sítio. Por outro lado, em idades mais avançadas, a distinção dessas classes apresenta-se mais evidente, sendo maior os limites das curvas de sítio. Nesse caso, a sensibilidade de mudança de classe de sítio às variações na altura dominante é mínima. Observações semelhantes realizadas por Silva et al. (2013), em classificação de sítio para Pinus caribaea var. hondurensis no Triângulo Mineiro, Minas Gerais.

Outros estudos também aplicaram este teste para analisar a forma das curvas de índices de sítio. Dentre estes, tem-se os trabalhos de Scolforo (1992), Machado et al. (1997), Téo et al. (2011) e Schuchovski et al. (2019). Os resultados deste teste indicaram o anamorfismo das curvas de índice de sítio.

Observando-se o resultado do teste proposto por DeMars (1969) (Figura 3b), ambos os métodos avaliados produziram curvas anamórficas, dada à sobreposição destas curvas. Isso evidencia forte proporcionalidade das curvas, desde as idades iniciais até aquelas mais avançadas.

O comportamento anamórfico de curvas de índices de sítio foi observado em outros trabalhos com o gênero em estudo. Como exemplos, o padrão anamórfico foi verificado por Scolforo (1992) para povoamentos de Pinus caribaea var. hondurensis localizados no Município de Agudos, São Paulo, utilizando o método da ED; Téo et al. (2011) em povoamentos de Pinus taeda L. na região de Caçador, Santa Catarina, por meio do método da CG; Silva et al. (2013) para plantios comerciais de Pinus caribaea var. hondurensis na região do Triângulo Mineiro, Minas Gerais, os quais empregaram o método da CG; Schuchovski et al. (2019) para povoamentos de Pinus taeda L. situados nos estados do Paraná, Santa Catarina e São Paulo, por meio do método da CG. 


\section{Desenvolvimento e avaliação de modelos hipsométricos}

De posse das estimativas de índices de sítio obtidas pelos métodos da CG e ED, os modelos hipsométricos genéricos foram ajustados. Conforme os valores das estatísticas de avaliação, para um mesmo modelo, os métodos da CG e ED não influenciaram expressivamente as estimativas de altura total (Tabela 3). Resultados similares foram obtidos aos dados destinados à validação, embora o método da ED tenha proporcionado estimativas pouco mais precisas, conforme os valores de RQMR (\%). Para todos os modelos de ambos os métodos de construção de curvas de índices de sítio em estudo, todos os coeficientes foram significativos, com destaque ao modelo 4 , seguido pelo modelo 3.

Tabela 3. Coeficientes e estatísticas de avaliação dos modelos testados para estimar altura total em um povoamento de Pinus taeda L.

\begin{tabular}{ccccccccc}
\hline \multirow{2}{*}{ Método } & Modelo & \multirow{2}{*}{$\widehat{\boldsymbol{\beta}}_{\mathbf{0}}$} & \multirow{2}{*}{$\widehat{\boldsymbol{\beta}}_{\mathbf{1}}$} & $\widehat{\boldsymbol{\beta}}_{\mathbf{2}}$ & $\widehat{\boldsymbol{\beta}}_{\mathbf{3}}$ & \multicolumn{2}{c}{ Ajuste } & \multicolumn{2}{c}{ Validação } \\
\cline { 7 - 9 } & & & & & & $\mathbf{R}^{\mathbf{2}}{ }_{\text {aj }}$ & $\mathbf{S}_{\mathbf{y x}}$ \% & RQMR (\%) \\
\hline \multirow{2}{*}{ CG } & 1 & $-10,78807$ & $-64,12279$ & 0,25196 & 10,60151 & 0,91 & 11,38 & 11,68 \\
& 2 & $-19,33847$ & 0,18481 & 4,66582 & 0,59133 & 0,93 & 9,87 & 9,92 \\
& 3 & $-3,78425$ & 1,02774 & 0,27619 & 0,12453 & 0,93 & 9,85 & 9,70 \\
& 4 & 1,21133 & 0,01048 & $-6,34359$ & 0,69040 & 0,94 & 9,40 & 9,16 \\
\multirow{2}{*}{ ED } & 1 & $-9,83849$ & $-64,14465$ & 0,27197 & 10,10742 & 0,91 & 11,34 & 11,63 \\
& 2 & $-18,92164$ & 0,20058 & 4,62128 & 0,55976 & 0,93 & 9,88 & 9,91 \\
& 3 & $-3,71111$ & 1,00152 & 0,27559 & 0,14018 & 0,93 & 9,82 & 9,65 \\
& 4 & 1,25243 & 0,01123 & $-6,34401$ & 0,66995 & 0,94 & 9,39 & 9,13 \\
\hline
\end{tabular}

Em que: $\mathrm{R}^{2}$ aj = coeficiente de determinação ajustado; $\mathrm{Sy}_{\mathrm{yx}} \%$ = erro padrão da estimativa percentual; RQMR (\%) = raiz do quadrado médio do resíduo percentual; $\hat{\beta}_{i}=$ coeficientes estimados.

A distribuição dos resíduos para a estimativa da altura total aos dados da validação foi observada para todos os modelos, contudo, a que mais se destacou foi aquela para o modelo 4 para os métodos da CG e ED. Essa distribuição demonstra forte similaridade dos resíduos, além de que ambos os métodos superestimaram a altura total, mas com baixa magnitude (Figura 4a). Cerca de 70\% das alturas foram superestimadas até os 8 anos, dada à maior variabilidade desta variável nas idades iniciais. A compatibilidade das estimativas de altura é corroborada pela Figura 4b, assim como pela forte correlação de Pearson, com valor de $r_{y \hat{y}}=0,9999$.
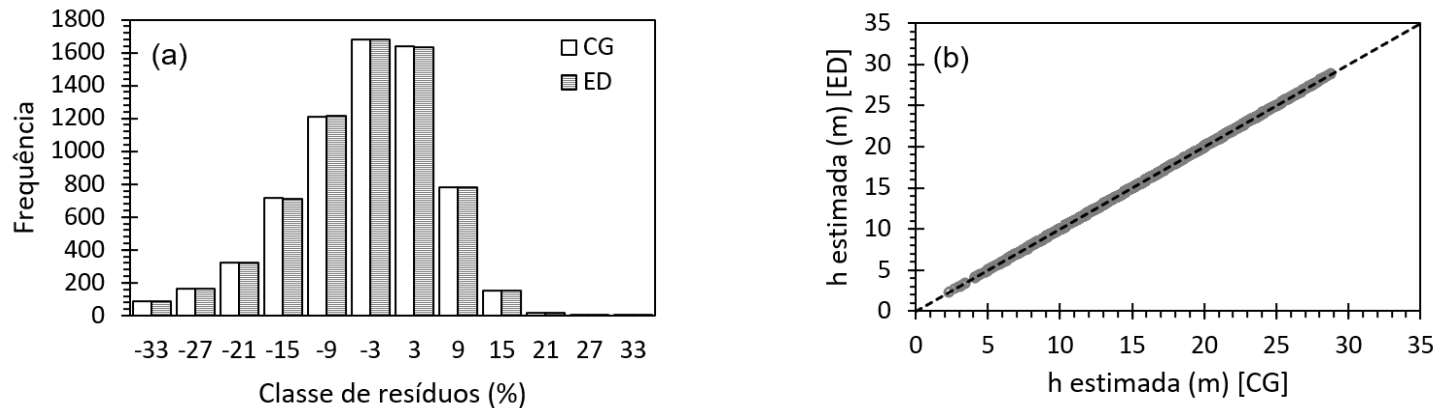

Figura 4. Distribuições dos resíduos para estimativa de altura total (a) e compatibilidade entre as estimativas de altura total para o modelo 4 por meios dos métodos da CG e ED (b), para povoamentos de Pinus taeda L.

O modelo 4 (Nogueira, 2003), utilizando tanto o índice de sítio do método da CG quanto do método da ED foi considerado o mais apropriado para estimar a altura total do povoamento em estudo. Como forma de verificar a eficácia deste modelo em estimar a altura 
total em diferentes idades e classes de sítio, a Figura 5 foi elaborada. As simulações foram realizadas considerando os índices de sítio pelos métodos da CG e ED. Como esperado, o modelo 4 produziu curvas dinâmicas com o avanço da idade, sem diferenciação da variável índice de sítio obtido pelos métodos da CG e ED. O modelo retratou satisfatoriamente a dinâmica da relação hipsométrica nas diferentes idades e nas três classes de índices de sítio. Comportamento semelhante foi observado por Juma et al. (2014) para Pinus patula Schltdl. \& Cham. na Estação Florestal de Londiani, na província de Kericho, Condado de Rift Valley, Quênia; e por Bartoszeck et al. (2004), para bracatingais nativos localizados na região metropolitana de Curitiba.
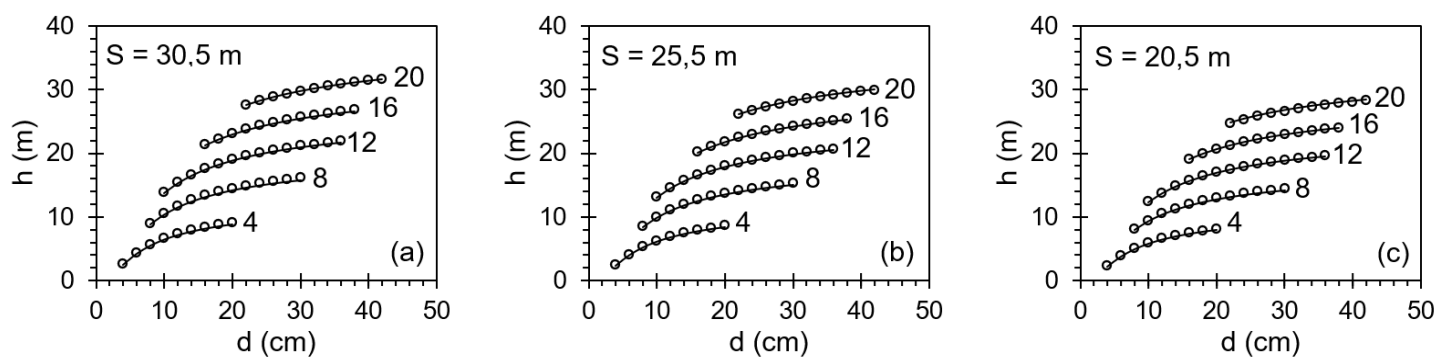

Figura 5. Predição da altura total em diferentes idades (4, 8, 12, 16 e 20 anos) com a variável independente índice de sítio obtida pelo método da CG (-) e ED (o), para as classes de sítios 30,5 m (a), 25,5 m (b) e 20,5 m (c), para povoamentos de Pinus taeda L.

Com base nos resultados, pode-se afirmar que a hipótese $h_{0(1)}$ foi rejeitada, uma vez que as estimativas de índice de sítio, obtidas pelos métodos da CG e ED, não foram estatisticamente compatíveis, cuja superioridade foi para o método da ED. A hipótese $\mathrm{h}_{0(2)}$ não pode ser rejeitada, pois os métodos da CG e ED promoveram estimativas de índice de sítio correlacionadas com a altura total e compatíveis entre si estatisticamente.

Métodos de classificação da capacidade produtiva são utilizados para determinação das classes de índices de sítio, permitindo associar o sítio específico a cada unidade amostral. A finalidade não é meramente realizar a distinção do povoamento em classes de produtividade, mas, acima de tudo, o sítio é um importante fator para estratificação e variável independente em modelos de crescimento e produção, seja para árvores individuais ou para o povoamento.

Apesar dos resultados terem indicado que os métodos de obtenção de índice de sítio não influenciaram as estimativas de altura total, este estudo ressalta a necessidade da avaliação do efeito da estimativa do sítio em todo o processo de modelagem florestal. Corroboram com isso Castro et al. (2015), os quais concluíram que o método de obtenção de curvas de índices de sítio exerceu influência na produção volumétrica em nível de povoamento em clones de Eucalyptus grandis W. Hill ex Maiden x Eucalyptus urophylla S. T. Blake, localizados na região de Santa Bárbara, Minas Grais.

\section{CONCLUSÃO}

Os métodos da curva-guia e equação das diferenças estimam índices de sítio diferentes estatisticamente. Os testes para analisar a forma das curvas indicaram que a relação altura dominante e idade podem ser representadas por curvas anamórficas. Entretanto, recomenda-se o método da equação das diferenças por sua flexibilidade em expressar o polimorfismo das curvas, o que está diretamente relacionado ao padrão de crescimento das árvores do povoamento. As curvas construídas pelo método da equação das diferenças apresentaram curvas mais estáveis do que o método da curva-guia, todavia, os índices de sítio estimados por ambos os métodos, inseridos nos modelos hipsométricos, promoveram estimativas compatíveis estatisticamente e acuradas da altura total. 


\section{REFERÊNCIAS BIBLIOGRÁFICAS}

Akbas, U., \& Senyurt, M. (2018). Site quality estimations based on the generalized algebraic difference approach: a case study in Çankiri forests. Árvore, 42(3), e420311. http://dx.doi.org/10.1590/180690882018000300011.

Assmann, E. (1970). The principles of forest yield study: studies in the organic production, structure, increment and yield of forest stands (506 p.). Oxford: Pergamon Press.

Bartoszeck, A. C. P. S., Machado, S. A., Figueiredo Filho, A., \& Oliveira, E. B. (2004). Dinâmica da relação hipsométrica em função da idade, do sítio e da densidade inicial de povoamentos de bracatinga da Região Metropolitana de Curitiba, PR. Árvore, 28(4), 517-533. http://dx.doi.org/10.1590/S010067622004000400006.

Attis Beltrán, H., Chauchard, L. M., \& Martínez Pastur, G. (2015). Curvas preliminares de índice de sitio para bosques puros y mixtos de Nothofagus alpina y Nothofagus obliqua en la Patagonia Argentina. Bosque (Valdivia), 36(2), 275-285. http://dx.doi.org/10.4067/S0717-92002015000200013.

Campos, J. C. C., \& Leite, H. G. (2017). Mensuração florestal: perguntas e respostas (5. ed., 636 p.). Viçosa: Editora UFV.

Castro, R. V. O., Cunha, A. B., Silva, L. V., Leite, H. G., \& Silva, A. A. L. (2015). Modelagem do crescimento e produção para um povoamento de Eucalyptus utilizando dois métodos para quantificação do índice de local. Scientia Forestalis, 43(105), 83-90.

Climate-Data.Org. (2021). Dados climáticos para cidades mundiais. Recuperado em 22 de março de 2021, de https://pt.climate-data.org/america-do-sul/brasil/parana/telemaco-borba-765176/

DeMars, D. J. (1969). Polymorphic site index curves for noble fir from stem analysis data (Thesis master). Oregon State University, Oregon.

Dias, A. N., Leite, H. G., Nogueira, G. S., \& Rodrigues, F. L. (2005). Avaliação de métodos de ajuste de curvas de índices de local em povoamentos de eucalipto desbastados. Árvore, 29(5), 741-747.

Draper, N. R., \& Smith, H. (1998). Applied regression analysis (3. ed., 706 p.). New York: John Wiley \& Sons.

Figueiredo, E. O. (2005). Avaliação de modelos pelo método da curva média para a construção de curvas de índice de sítio para Tectona grandis L. f. (No. 42, Boletim de Pesquisa e Desenvolvimento, 49 p.). Rio Branco, AC: Embrapa Acre.

Hammer, G. L. (1981). Site classification and tree diameter-height-age relationships for cypress pine in the top end of the northern territory. Australian Forestry, 44(1), 35-41. http://dx.doi.org/10.1080/00049158.1981.10674287.

Juma, R., Pukkala, T., de-Miguel, S., \& Muchiri, M. (2014). Evaluation of different approaches to individual tree growth and survival modelling using data collected at irregular intervals - a case study for Pinus patula in Kenya. Forest Ecosystems, 1(14), 14. http://dx.doi.org/10.1186/PREACCEPT9097767721272407.

Kirby, C. L. (1975). Site index equations for lodgepole pine and white pine in Alberta (12 p.). Edmonton: Northern Forest Research Centre.

Machado, S. A., Oliveira, E. B., Carpanezzi, A. A., \& Bartoszeck, A. C. P. S. (1997). Classificação de sítio para bracatingais na região metropolitana de Curitiba. Boletim de Pesquisa Florestal, 35, 21-37.

Nogueira, G. S. (2003). Modelagem do crescimento e da produção de povoamentos de Eucalyptus sp. e de Tectona grandis submetidas a desbaste (Tese de doutorado). Universidade Federal de Viçosa, Viçosa.

Nunes, L., Patricio, M., Tomé, J., \& Tomé, M. (2011). Modeling dominant height growth of maritime pine in Portugal using GADA methodology with parameters depending on soil and climate variables. Annals of Forest Science, 68(2), 311-323. http://dx.doi.org/10.1007/s13595-011-0036-8.

Pego, M. F. F., Assis, A. L., \& Cabacinha, C. D. (2015). Classificação de sítios florestais em povoamentos de eucalipto na microrregião de Salinas, Minas Gerais. Enciclopédia Biosfera, 11(21), 534-542.

Regazzi, A. J. (2003). Teste para verificar a igualdade de parâmetros e a identidade de modelos de regressão não linear. Ceres, 50(287), 9-26.

Retslaff, F. A. S., Figueiredo Filho, A., Dias, A. N., Bernett, L. G., \& Figura, M. A. (2015). Curvas de sítio e relações hipsométricas para Eucalyptus grandis na região dos Campos Gerais, Paraná. Cerne, 21(2), 219-225. http://dx.doi.org/10.1590/01047760201521021349.

Santos, C. J., Thiersch, C. R., \& Moreira, M. F. B. (2018). Regressão não linear quantílica para classificação de sítio em povoamentos de Eucalyptus sp. Scientia Forestalis, 46(120), 551-561. http://dx.doi.org/10.18671/scifor.v46n120.04. 
Schuchovski, M. S., Arce, J. E., \& Oliveira, E. B. (2019). Altura dominante e índice de sítio para Pinus taeda em dois polos de desenvolvimento florestal no sul do Brasil. Scientia Forestalis, 47(121), 92-104. http://dx.doi.org/10.18671/scifor.v47n121.09.

Scolforo, J. R. S. (2006). Biometria florestal: modelos de crescimento e produção florestal (393 p.). Lavras: FAEPE-UFLA.

Scolforo, J. R. S. (1992). Curvas de índice de sítio para Pinus caribaea var. hondurensis. IPEF, 45, 40-47.

Selle, G. L., Pauleski, D. T., \& Braz, E. M. (2008). Como classificar sítios florestais através da altura dominante do povoamento (Documentos, No. 166, 46 p.). Colombo: Embrapa Florestas.

Selle, G. L., Schneider, P. R., \& Finger, C. A. G. (1994). Classificação de sítio para Pinus taeda L., através da altura dominante, para a região de Cambará do Sul, RS, Brasil. Ciência Florestal, 4(1), 77-95. http://dx.doi.org/10.5902/19805098299.

Silva, F., Pimentel, A., Corte, A. P. D., \& Sanquetta, C. R. (2013). Classificação de sítio para Pinus caribaea var. hondurensis na região do Triângulo Mineiro. Revista Acadêmica Ciências Agrárias e Ambientais, 11(Supl. 1), S105-S112. http://dx.doi.org/10.7213/academica.10.S01.A012.

Silva, F. R., Silva, V. S. M., Miranda, S. O., Oliveira, B. R., \& Silva, J. C. (2016). Classificação da produtividade para um plantio de Tectona grandis em Alta Floresta, Mato Grosso. Ambiência, 12(4), 809-823. http://dx.doi.org/10.5935/ambiencia.2016.04.04.

Skovsgaard, J. P., \& Vanclay, J. K. (2008). Forest site productivity: a review of the evolution of dendrometric concepts for even-aged stands. Forestry, 81(1), 13-31. http://dx.doi.org/10.1093/forestry/cpm041.

Téo, S. J., Bressan, D. R., \& Costa, R. H. (2011). Uso de modelos estatísticos para classificação de sítios em povoamentos de Pinus taeda na região de Caçador, SC. Floresta, 41(1), 179-188. http://dx.doi.org/10.5380/rf.v41i1.21201.

Tonini, H., Schneider, P. R., \& Finger, C. A. G. (2006). Curvas de índice de sítio para povoamentos clonais de Eucalyptus saligna Smith para a Depressão Central e Serra do Sudeste, Rio Grande do Sul. Ciência Florestal, 16(1), 27-43. http://dx.doi.org/10.5902/198050981885.

Zeide, B. (1993). Analysis of growth equations. Forest Science, 39(3), 594-616. http://dx.doi.org/10.1093/forestscience/39.3.594.

Contribuição dos Autores: ROVM: conceituação, curadoria de dados, análise formal, investigação, metodologia, visualização, escrita - primeira redação, escrita - revisão e edição; AFF: conceituação, investigação, metodologia, supervisão, escrita - primeira redação; EAC: análise formal, metodologia, visualização, escrita - revisão e edição; LDF: análise formal, validação, visualização; SVK: investigação, visualização, escrita - primeira redação; AAE: análise formal, escrita - primeira redação, escrita - revisão e edição. 